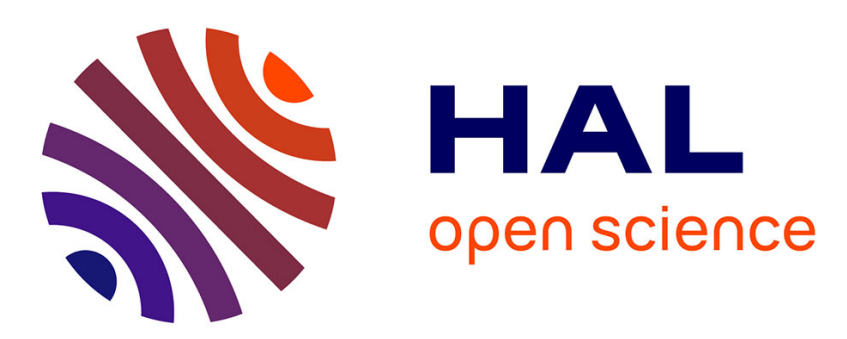

\title{
Exactly solvable one-dimensional inhomogeneous models
}

\author{
Bernard Derrida, M. Mendès France, J. Peyrière
}

\section{To cite this version:}

Bernard Derrida, M. Mendès France, J. Peyrière. Exactly solvable one-dimensional inhomogeneous models. Journal of Statistical Physics, 1986, 45 (3-4), pp.439-449. 10.1007/BF01021080 . hal03285913

\section{HAL Id: hal-03285913 \\ https://hal.science/hal-03285913}

Submitted on 19 Jul 2021

HAL is a multi-disciplinary open access archive for the deposit and dissemination of scientific research documents, whether they are published or not. The documents may come from teaching and research institutions in France or abroad, or from public or private research centers.
L'archive ouverte pluridisciplinaire HAL, est destinée au dépôt et à la diffusion de documents scientifiques de niveau recherche, publiés ou non, émanant des établissements d'enseignement et de recherche français ou étrangers, des laboratoires publics ou privés. 


\title{
Exactly Solvable One-Dimensional Inhomogeneous Models
}

\author{
B. Derrida, ${ }^{1}$ M. Mendès France, ${ }^{2}$ and J. Peyrière ${ }^{3}$
}

Received April 28, 1986; revision received July 25, 1986

\begin{abstract}
We present a simple way of constructing one-dimensional inhomogeneous models (random or quasiperiodic) which can be solved exactly. We treat the example of an Ising chain in a varying magnetic field, but our procedure can easily be extended to other one-dimensional inhomogeneous models. For all the models we can construct, the free energy and its derivatives with respect to temperature can be computed exactly at one particular temperature.
\end{abstract}

KEY WORDS: Liapunov exponent; Ising chain; disordered chain; quasiperiodic chain; exactly solvable model.

\section{INTRODUCTION}

One-dimensional models in classical statistical mechanics are known to be exactly and easily solvable. However, as soon as the one-dimensional model becomes inhomogeneous, ${ }^{(1)}$ i.e., the parameters that define the model (magnetic field or nearest neighbor interactions for Ising models, potential in the Anderson model, etc.) vary in space (random models or quasiperiodic models), finding the exact solution usually becomes next to impossible except in a few special examples ${ }^{(2)}$ or in some limiting cases $^{(3,4)}$ (zero temperature, weak disorder,...).

In the present work, we show how to construct a large class of inhomogeneous models (random or quasiperiodic) for which the free energy (or Liapunov exponent) can be computed exactly. In order to illustrate our method, we restrict our discussion to an Ising chain with a

\footnotetext{
' Service de Physique Théorique, CEN Saclay, 91191 Gif sur Yvette, France.

${ }^{2}$ Département de Mathématiques et Informatique, Université de Bordeaux 1, 33405 Talence, France.

${ }^{3}$ Département de Mathématiques, Université de Paris-Sud, 91405 Orsay, France.
} 
varying external field. Our method can nevertheless be easily extended to Ising chains with nonconstant interactions in a varying field, to the problem of diffusion in inhomogeneous media, to the problem of a quantum particle in a varying potential, etc.

Our approach is rather artificial because we start by giving the solution and then build the model knowing its solution. As a consequence, we compute the free energy and its derivatives at a specific temperature only. The calculation of the $n$th derivative with respect to temperature is possible. Yet the complexity increases rapidly with the order of derivation. The knowledge of the free energy at a single point is very reminiscent of some spin models for which the partition function can be computed exactly on some special lines in a phase diagram (the so-called disordered lines) ${ }^{(5)}$ and also of the exact results known on the Nishimori line in spin glasses. ${ }^{(6)}$

In Section 6 we compare our work to that of Percus, ${ }^{(7)}$ who computes the site-dependent magnetic field that produces a given magnetization profile.

\section{SOLVING THE MODEL}

Consider an Ising chain in a variable external field $h_{i}$. Its Hamiltonian is

$$
\mathscr{H}(\sigma)=-\sum_{i=1}^{N-1} J \sigma_{i} \sigma_{i+1}-\sum_{i=1}^{N} h_{i} \sigma_{i}
$$

where as usual $\sigma_{i}$ denotes the Ising spin $\left(\sigma_{i}= \pm 1\right)$ at site $i$ and $J$ is the coupling constant. For certain choices of the $h_{i}$ we shall see that we can compute the free energy exactly.

Let

$$
Z_{N}=\sum_{\sigma \in\{-1,1\}^{N}} \exp [-\mathscr{H}(\sigma)]
$$

be the partition function (notice we have normalized the temperature $T=1$ ). The easiest way to calculate $Z_{N}$ is to use the transfer matrix technique, which consists in expressing $Z_{n+1}$ in terms of $Z_{n}$. To be more specific, define $Z_{n}(+)$ to be the partition function of the Ising chain starting at site 1 , ending at site $n$, with the extra condition $\sigma_{n}=+1$. In the same fashion, we define $Z_{n}(-)$ with $\sigma_{n}=-1$. Hence, for $\varepsilon= \pm$,

$$
Z_{n}(\varepsilon)=\sum_{\sigma \in\{-1,1\}^{n-1}} \exp \left(J \sum_{i=1}^{n-2} \sigma_{i} \sigma_{i+1}+J \varepsilon \sigma_{n-1}+\sum_{i=1}^{n-1} h_{i} \sigma_{i}+\varepsilon h_{n}\right)
$$


It is easy to see that

$$
\begin{aligned}
& Z_{n+1}(+)=e^{h_{n+1}}\left[e^{J} Z_{n}(+)+e^{-J} Z_{n}(-)\right] \\
& Z_{n+1}(-)=e^{-h_{n+1}}\left[e^{-J} Z_{n}(+)+e^{J} Z_{n}(-)\right]
\end{aligned}
$$

Put

$$
r_{n}=Z_{n}(+) / Z_{n}(-)
$$

The above recurrence yields

$$
r_{n+1}=e^{2 h_{n+1}} \frac{e^{2 J} r_{n}+1}{r_{n}+e^{2 J}}
$$

Usually one is asked to compute the $r_{n}$, given the $h_{n}$. Indeed, the free energy $\psi$ per spin is expressed in terms of the $r_{n}$ in the following way:

$$
\begin{aligned}
-\psi & =\lim _{N \rightarrow \infty} \frac{1}{N} \log Z_{N} \\
& =\lim _{N \rightarrow \infty} \frac{1}{N} \log \left[Z_{N}(+)+Z_{N}(-)\right] \\
& =\lim _{N \rightarrow \infty}\left\{\frac{1}{N} \sum_{n=2}^{N} \log \left(\frac{Z_{n}(+)+Z_{n}(-)}{Z_{n-1}(+)+Z_{n-1}(-)}\right)+\frac{1}{N} \log \left[Z_{1}(+)+Z_{1}(-)\right]\right\} \\
& =\lim _{N \rightarrow \infty}\left\{\frac{1}{N} \sum_{n=2}^{N} \log \left[e^{-h_{n}}\left(e^{-J} r_{n-1}+e^{J}\right)\right]+\frac{1}{N} \log \frac{r_{N}+1}{r_{1}+1}\right\}
\end{aligned}
$$

We now make the assumption that as $N$ increases to infinity, $r_{N}$ is bounded. This is of course the common case. Then, using (2), we have

$$
\begin{aligned}
-\psi & =\lim _{N \rightarrow \infty} \frac{1}{N} \sum_{n=1}^{N} \log \left[\left(e^{-J} r_{n-1}+e^{J}\right) e^{-h_{n}}\right] \\
& =\lim _{N \rightarrow \infty} \frac{1}{N} \sum_{n=1}^{N} \frac{1}{2} \log \frac{\left(e^{J}+e^{-J} r_{n-1}\right)\left(e^{-J}+e^{J} r_{n-1}\right)}{r_{n}} \\
& =\frac{1}{2} \lim _{N \rightarrow \infty} \frac{1}{N} \sum_{n=1}^{N} \log \frac{1+2 r_{n-1} \cosh 2 J+r_{n-1}^{2}}{r_{n}}
\end{aligned}
$$

Notice that

$$
\lim _{N \rightarrow \infty} \frac{1}{N} \sum_{n=1}^{N} \log \frac{1}{r_{n}}=\lim _{N \rightarrow \infty} \frac{1}{N} \sum_{n=2}^{N} \log \frac{1}{r_{n-1}}
$$


Thence

$$
-\psi=\frac{1}{2} \lim _{N \rightarrow \infty} \frac{1}{N} \sum_{n=1}^{N} \log \left(r_{n}+\frac{1}{r_{n}}+2 \cosh 2 J\right)
$$

This calculation is valid provided the limits exist.

In the usual approach, it is difficult to compute the free energy because the sequence $r_{n}$ is defined through the recurrence relation (2). If $h_{n}$ is not constant, $r_{n}$ can be too complicated to allow the calculation of the free energy using $(3 b)$. We propose here to solve the problem from the "other end." We are given the sequence $r_{n}$. We then obtain both the external field $h_{n}$ from formula (2)

$$
e^{2 h_{n}}=r_{n} \frac{r_{n-1}+e^{2 J}}{e^{2 J} r_{n-1}+1}
$$

and the free energy $\psi$ from formula (3b). We illustrate this principle in the next Section, leaving the discussion of the derivatives of $\psi$ to Section 5.

\section{A GENERIC EXAMPLE}

Let us first recall a useful result due to $\mathrm{H}$. Weyl (see Ref. 8).

Lemma. If $\left(u_{n}\right)$ is an equidistributed sequence $(\bmod 1)$, then for all Riemann integrable functions $\Phi$ with period 1

$$
\lim _{N \rightarrow \infty} \frac{1}{N} \sum_{n=1}^{N} \Phi\left(u_{n}\right)=\int_{0}^{1} \Phi(x) d x
$$

Examples of such sequences are $u_{n}=n \alpha$ ( $\alpha$ irrational), $u_{n}=n^{2} \alpha(\alpha$ irrational), $u_{n}=\sqrt{n}$, etc., As for Riemann integrable functions, any real periodic continuous functions with period 1 will suffice for our applications.

Suppose we are given an equidistributed sequence $(\bmod 1)$, say $u_{n}$, and a continuous function $\varphi$ with period 1 . We choose $r_{n}=\varphi\left(u_{n}\right)$. In order to give a physical meaning to $r_{n}$, we must assume $\varphi>0$ [because $r_{n}$ is the ratio of two positive numbers; Eq. (1)]. We shall actually demand slightly more, namely that there exists a constant $C>0$ such that for all $x \in(0,1)$, $\varphi(x)>C$. Apply Weyl's lemma to

$$
\Phi(x)=\log \left[\varphi(x)+\frac{1}{\varphi(x)}+2 \cosh 2 J\right]
$$

Then the free energy is given by

$$
-\psi=\frac{1}{2} \int_{0}^{1} \log \left[\varphi(x)+\frac{1}{\varphi(x)}+2 \cosh 2 J\right] d x
$$




\section{QUASIPERIODIC AND RANDOM EXAMPLES}

One can choose for $u_{n}$ a quasiperiodic sequence, say $u_{n}=(n+1) \alpha$, where $\alpha$ is irrational. For example, take

$$
r_{n}=\lambda_{n}+\left(\lambda_{n}^{2}-1\right)^{1 / 2}=\varphi\left(u_{n}\right)
$$

where

$$
\lambda_{n}=A+B \cos 2 \pi(n+1) \alpha, \quad 0<B<A-1, \quad \alpha \text { irrational }
$$

The sequence $r_{n}$ is quasiperiodic.

The field $h_{n}$ is then given by (4). More specifically

$$
\begin{aligned}
h_{n}= & \frac{1}{2}\left[\log \left(A+B \cos 2 \pi(n+1) \alpha+\left\{[A+B \cos 2 \pi(n+1) \alpha]^{2}-1\right\}^{1 / 2}\right)\right. \\
& +\log \left\{e^{2 J}+A+B \cos 2 \pi n \alpha+\left[(A+B \cos 2 \pi n \alpha)^{2}-1\right]^{1 / 2}\right\} \\
& -\log \left\{1+A e^{2 J}+B e^{2 J} \cos 2 \pi n \alpha\right. \\
& \left.\left.+e^{2 J}\left[(A+B \cos 2 \pi n \alpha)^{2}-1\right]^{1 / 2}\right\}\right]
\end{aligned}
$$

The field $h_{n}$ is seen to be also quasiperiodic. A simple calculation using formula (5) leads to

$$
-\psi=\frac{1}{2} \log \left\{A+\cosh 2 J+\left[(A+\cosh 2 J)^{2}-B^{2}\right]^{1 / 2}\right\}
$$

We thus conclude that for a quasiperiodic field $h_{n}$ given by (6), the free energy can be computed exactly and the result is (7).

In the previous example, $r_{n}$ and $h_{n}$ are deterministic sequences. In the same fashion one can construct random sequences. For example, if we choose

$$
\lambda_{n}=A+B \cos \left(2 \pi X_{n}\right)
$$

where $X_{n}$ is a uniformly distributed random variable on the interval $(0,1)$, then the field $h_{n}$ becomes random and is given by

$$
\begin{aligned}
h_{n}= & \frac{1}{2}\left(\log \left\{A+B \cos 2 \pi X_{n}+\left[\left(A+B \cos 2 \pi X_{n}\right)^{2}-1\right]^{1 / 2}\right\}\right. \\
& +\log \left\{e^{2 J}+A+B \cos 2 \pi X_{n-1}+\left[\left(A+B \cos 2 \pi X_{n-1}\right)^{2}-1\right]^{1 / 2}\right\} \\
& -\log \left\{1+A e^{2 J}+B e^{2 J} \cos 2 \pi X_{n-1}\right. \\
& \left.\left.+e^{2 J}\left[\left(A+B \cos 2 \pi X_{n-1}\right)^{2}-1\right]^{1 / 2}\right\}\right)
\end{aligned}
$$

The sequence $h_{n}$ is random, but $h_{n}$ is correlated to $h_{n-1}$ and to $h_{n+1}$ 
because $h_{n}$ and $h_{n-1}$ both depend on $X_{n-1}$. Observe, however, that if the $X_{n}$ are independent, there is no further correlation

$$
\left[\left\langle h_{n+p} h_{n}\right\rangle-\left\langle h_{n+p}\right\rangle\left\langle h_{n}\right\rangle=0 \text { for } p \geqslant 2 \text { or } p \leqslant-2\right]
$$

It is clear that for the random sequence (8), the free energy is again given by (7).

\section{DERIVATIVES OF THE FREE ENERGY}

We have seen in Sections 2 and 3 that, given the sequence $r_{n}$, the sequence $h_{n}$ is defined by

$$
h_{n}=\frac{1}{2} \log \frac{r_{n}\left(r_{n-1}+e^{2 J}\right)}{e^{2 J} r_{n-1}+1}
$$

and the free energy $\psi$ at $\beta=1$ is given by

$$
-\psi=\frac{1}{2} \lim _{N \rightarrow \infty} \frac{1}{N} \sum_{n=1}^{N} \log \left(\frac{1}{r_{n}}+r_{n}+2 \cosh 2 J\right)
$$

Suppose we want to compute the free energy of the chain where $h_{n}$ is defined by (9) at temperature $T=1 / \beta \neq 1$. We then have to compute $s_{n}$ defined by the recurrence

$$
s_{n+1}=e^{2 \beta h_{n+1}} \frac{e^{2 \beta J} s_{n}+1}{e^{2 \beta J}+s_{n}}
$$

As before, the free energy $\psi$ will be given by

$$
-\beta \psi=\frac{1}{2} \lim _{N \rightarrow \infty} \frac{1}{N} \sum_{n=1}^{N} \log \left(\frac{1}{s_{n}}+s_{n}+2 \cosh 2 \beta J\right)
$$

Obviously for $\beta=1$, one has $s_{n}=r_{n}$. Computing the derivatives of $\psi$ with respect to $\beta$ at $\beta=1$ involves computing the derivatives of $s_{n}$ at $\beta=1$. Let us show how to calculate $d \psi / d \beta$ :

$$
-\left.\frac{d(\beta \psi)}{d \beta}\right|_{\beta=1}=\lim _{N \rightarrow \infty} \frac{1}{N} \sum_{n=1}^{N}\left[d\left(r_{n}\right)+\left.c\left(r_{n}\right) \frac{s_{n}^{\prime}}{s_{n}}\right|_{\beta=1}\right]
$$

where

$$
\begin{aligned}
& c\left(r_{n}\right)=\frac{1}{2} \frac{r_{n}^{2}-1}{r_{n}^{2}+1+2 r_{n} \cosh 2 J} \\
& d\left(r_{n}\right)=\frac{2 J r_{n} \sinh 2 J}{r_{n}^{2}+1+2 r_{n} \cosh 2 J}
\end{aligned}
$$


The careful reader may well criticize the liberty with which we inverted the limit operator and the derivation. For each choice of the sequence $r_{n}$, one should actually justify this step. However, at this stage we wish only to present a method and we disregard this mathematical technicality.

Formula (13) shows that the calculation of $d \psi / d \beta$ requires the calculation of $s_{n}^{\prime} / s_{n}$ at $\beta=1$ only. Formula (11) implies the following induction formula, which relates $s_{n+1}^{\prime} / s_{n+1}$ to $s_{n}^{\prime} / s_{n}$ :

$$
\frac{s_{n+1}^{\prime}}{s_{n+1}}=a\left(r_{n}\right) \frac{s_{n}^{\prime}}{s_{n}}+b\left(r_{n+1}, r_{n}\right)
$$

where

$$
\begin{gathered}
a\left(r_{n}\right)=r_{n}\left(\frac{e^{2 J}}{e^{2 J} r_{n}+1}-\frac{1}{e^{2 J}+r_{n}}\right) \\
b\left(r_{n+1}, r_{n}\right)=2 h_{n+1}+2 J\left(\frac{e^{2 J} r_{n}}{e^{2 J} r_{n}+1}-\frac{e^{2 J}}{r_{n}+e^{2 J}}\right)
\end{gathered}
$$

Since the $r_{n}$ are positive, it is easily checked that

$$
\left|a\left(r_{n}\right)\right| \leqslant|\tanh J|<1
$$

Therefore, one can solve (15) easily:

$$
\frac{s_{n+1}^{\prime}}{s_{n+1}}=b\left(r_{n+1}, r_{n}\right)+\sum_{p=0}^{\infty}\left[\prod_{q=0}^{p} a\left(r_{n-q}\right)\right] b\left(r_{n-p}, r_{n \cdots p-1}\right)
$$

Going back to (13), one finds

$$
-\left.\frac{d(\beta \psi)}{d \beta}\right|_{\beta=1}=X+Y+\sum_{p=1}^{\infty} V_{p}
$$

where

$$
\begin{aligned}
X & =\lim _{N \rightarrow \infty} \frac{1}{N} \sum_{n=1}^{N} d\left(r_{n}\right) \\
Y & =\lim _{N \rightarrow \infty} \frac{1}{N} \sum_{n=1}^{N} c\left(r_{n}\right) b\left(r_{n+1}, r_{n}\right) \\
V_{p} & =\lim _{N \rightarrow \infty} \frac{1}{N} \sum_{n=1}^{N} c\left(r_{n}\right)\left[\prod_{q=1}^{p} a\left(r_{n-4}\right)\right] b\left(r_{n-p}, r_{n-p-1}\right)
\end{aligned}
$$

Remembering that the sequence $r_{n}$ is given, it is now left to compute $X, Y$, and $V_{p}$ either by Weyl's theorem if $r_{n}$ is uniformly distributed modulo 1 or 
by the strong law of large numbers if the $r_{n}$ are random variables. More specifically, if $r_{n}=\varphi(n \alpha), \alpha$ irrational, then

$$
\begin{aligned}
X & =\int_{0}^{1} d(\varphi(x)) d x \\
Y & =\int_{0}^{1} c(\varphi(x)) b(\varphi(x+\alpha), \varphi(x)) d x \\
V_{p} & =\int_{0}^{1} c(\varphi(x))\left[\prod_{q=0}^{p} a(\varphi(x-q \alpha))\right] b(\varphi(x-p \alpha), \varphi(x-p \alpha-\alpha)) d x
\end{aligned}
$$

and if $r_{n}$ are independent random variables uniformly distributed on $(0,1)$, $X$ is still given by (22), whereas

$$
\begin{aligned}
Y= & \int_{0}^{1} \int_{0}^{1} c(\varphi(x)) b(\varphi(y), \varphi(x)) d x d y \\
V_{p}= & {\left[\int_{0}^{1} c(\varphi(x)) d x\right]\left[\int_{0}^{1} a(\varphi(x)) d x\right]^{p-1} } \\
& \times\left[\int_{0}^{1} \int_{0}^{1} a(\varphi(x)) b(\varphi(y), \varphi(x)) d x d y\right]
\end{aligned}
$$

Notice that in this last case, the series $\sum_{p} V_{p}$ is a geometric convergent series.

Higher derivatives could be calculated in the same fashion. It goes without saying that their expression is more complex, because it involves higher derivatives of $s_{n}$. This is of course unfortunate. Had we been able to compute the derivatives, Taylor's formula would have given us the expression of the free energy at all temperatures.

The reason the construction of solvable inhomogeneous models is possible is rather obvious. It is clear that for these models, if one changes the temperature or any other physical parameter, the solution described above no longer holds. However, we believe that it is interesting to dispose of a class of models for which the free energy (or the Liapunov exponent) and its derivatives can be known exactly, though it is only known at a single temperature.

It is also clear that by choosing a more complicated sequence $r_{n}$ one can obtain sequences $h_{n}$ that may look less bizarre than (6) or (8) (the sequence $r_{n}$ we used in Section 4 was chosen in order to make the calculation of $\psi$ as simple as possible).

It would be interesting to see whether the very simple argument used here could be generalized to construct inhomogeneous models which would 
remain solvable at more than one temperature. A first step in that direction was discussed in the previous section. It would also be interesting to generalize the above argument to transfer matrices of size larger than 2 in order to treat the case of inhomogeneous models on strips.

\section{COMPARISON WITH THE METHOD OF PERCUS ${ }^{(7)}$}

The difference between our approach and that of Percus is that we are given the parameters $r_{n}=Z_{n}^{(+)} / Z_{n}^{(-)}$, whereas he is given the more physical quantities $m_{n}=\left(\partial / \partial h_{n}\right) \log Z_{n}$, which represent the magnetization at site $n$ (mean spin). We express the physical characteristics of the model in terms of $r_{n}$ and Percus in terms of $m_{n}$.

Now, even though the magnetization $m_{n}$ has a more physical meaning than $r_{n}$, there seems to be some gain in considering $r_{n}$. For instance, on the one hand, Percus notices that the external field $h_{n}$ is a function of the three variables $m_{n-1}, m_{n}$, and $m_{n+1}$ [see his equality, ${ }^{(7)}$ p. 303, relating $u(x)$, $\left.\sigma_{x-1}, \sigma_{x}, \sigma_{x+1}\right]$. On the other hand, our Eq. (4) shows that $h_{n}$ is a function of two variables only, $r_{n-1}$ and $r_{n}$. We have reduced the dimension.

The two approaches are nevertheless consistent with one another. To see this, it suffices to prove that $r_{n}$ is a function of $m_{n}$ and $m_{n+1}$. Indeed, recall formulas (2) and (3a):

$$
\begin{aligned}
r_{n+1} & =e^{2 h_{n+1}} \frac{e^{2 J} r_{n}+1}{r_{n}+e^{2 J}} \\
\log Z_{N} & =\sum_{n=2}^{N} \log \left[e^{-h_{n}}\left(e^{-J} r_{n-1}+e^{J}\right)\right]+\log \frac{r_{N}+1}{r_{1}+1}
\end{aligned}
$$

We now compute the magnetization,

$$
m_{n}=\frac{\partial}{\partial h_{n}} \log Z_{N}
$$

If we consider the external field $h_{n}$ as given, then formula (2) shows that $r_{n}$ is a function of $h_{1}, h_{2}, \ldots, h_{n}$. Hence, in the thermodynamic limit

$$
\begin{aligned}
m_{n} & =-1+\sum_{i=0}^{\infty} \frac{1}{r_{n+i}+e^{2 J}} \frac{\partial r_{n+i}}{\partial h_{n}} \\
m_{n+1} & =-1+\sum_{i=1}^{\infty} \frac{1}{r_{n+i}+e^{2 J}} \frac{\partial r_{n+i}}{\partial h_{n+1}}
\end{aligned}
$$


Put

$$
S=\sum_{i=1}^{\infty} \frac{1}{r_{n+i}+e^{2 J}} \frac{\partial r_{n+i}}{\partial r_{n+1}}
$$

Then

$$
m_{n}=-1+S \frac{\partial r_{n+1}}{\partial h_{n}}+\frac{1}{r_{n}+e^{2 J}} \frac{\partial r_{n}}{\partial h_{n}}
$$

and

$$
m_{n+1}=-1+S \frac{\partial r_{n+1}}{\partial h_{n+1}}
$$

We now wish to eliminate $S$ between $m_{n}$ and $m_{n+1}$. First note that

$$
\partial r_{n} / \partial h_{n}=2 r_{n}
$$

and

$$
\frac{\partial r_{n+1}}{\partial h_{n}}=e^{2 h_{n+1}} \frac{e^{4 J}-1}{\left(r_{n}+e^{2 J}\right)^{2}}=r_{n+1} \frac{e^{4 J}-1}{\left(r_{n}+e^{2 J}\right)\left(e^{2 J} r_{n}+1\right)}
$$

Hence

$$
\begin{aligned}
m_{n+1} & =-1+2 r_{n+1} S \\
m_{n} & =-1+2 r_{n}\left[r_{n+1} \frac{e^{4 J}-1}{\left(r_{n}+e^{2 J}\right)\left(e^{2 J} r_{n}+1\right)} S+\frac{1}{r_{n}+e^{2 J}}\right]
\end{aligned}
$$

We finally eliminate $S$ :

$$
m_{n}=-1+2 r_{n}\left[\frac{1+m_{n+1}}{2} \frac{e^{4 J}-1}{\left(r_{n}+e^{2 J}\right)\left(e^{2 J} r_{n}+1\right)}+\frac{1}{r_{n}+e^{2 J}}\right]
$$

This rather clumsy expression relates $r_{n}$ to $m_{n}$ and $m_{n+1}$ and establishes our claim.

Notice, however, that the expression for $r_{n}$ in terms of $m_{n}$ and $m_{n+1}$ involves a second-degree equation, which seems quite unwieldy. The relationship between our point of view and that of Percus, even though clear, appears to be too complicated to allow a mere translation from one system to another. 


\section{REFERENCES}

1. F. J. Dyson, Phys. Rev. 92:1331 (1953); H. Schmidt, Phys. Rev. 105:425 (1957); S. Alexander, J. Bernasconi, W. R. Schneider, and R. Orbach, Rev. Mod. Phys. 53:175 (1981); R. Bruinsma and G. Aeppli, Phys. Rev. Lett. 50:1495 (1983).

2. B. I. Halperin, Phys. Rev. A 139:104 (1965); P. Lloyd, J. Phys. C 2:1717 (1969); G. Grinstein and D. Mukamel, Phys. Rev. B 27:4503 (1983); R. A. Pelcovits and D. Mukamel, Phys. Rev. B 28:5374 (1983); T. M. Nieuwenhuizen, Analytic methods and exact solutions for one dimensional random systems, Thesis, Utrecht University (1983); J. Bernasconi and W. R. Schneider, Helv. Phys. Acta 58:597 (1985); J. A. Reger and K. Binder, Z. Phys. B 60:137 (1985); T. M. Nieuwenhuizen and J. M. Luck, J. Phys. A 19:1207 (1986).

3. A. Vilenkin, Phys. Rev. B 18:1474 (1978); V. Brandt and W. Gross, Z. Phys. B 31:237 (1978); B. Derrida, J. Vannimenus, and Y. Pomeau, J. Phys. C 11:4749 (1978); B. Derrida and H. J. Hilhorst, J. Phys. A 16:2641 (1983); E. Gardner and B. Derrida, J. Stat. Phys. 39:367 (1985); B. Derrida and A. Zippelius, J. Phys. (Paris) 47:955 (1986); J. P. Allouche and M. Mendès France, J. Stat. Phys. 42:809 (1986); M. Mendès France, J. Stat. Phys. 45:89 (1986).

4. M. Kappus and F. Wegner, Z. Phys. B 45:15 (1981); C. J. Lambert, Phys. Rev. B 29:1091 (1984); B. Derrida and E. Gardner, J. Phys. (Paris) 45:1283 (1984); A. Mielke and F. Wegner, Z. Phys. B 62:1 (1985); J. M. Luck and D. Petritis, J. Stat. Phys. 42:289 (1986).

5. J. Stephenson, J. Math. Phys. 11:420 (1970); I. G. Enting, J. Phys. A 10:1023 (1977); M. T. Jackel and J. M. Maillard, J. Phys. A 18:641, 1229, 2271 (1985).

6. H. Nishimori J. Phys. C 13:4071 (1980); Prog. Theor. Phys. 66:1169 (1981); H. Nishimori and M. J. Stephen, Phys. Rev. B 27:5644 (1983); A. Georges, D. Hansel, and P. Le Doussal, J. Phys. (Paris) 46:1309 (1985); A. Georges, D. Hansel, P. Le Doussal, and J. P. Bouchaud, J. Phys. (Paris) 46:1827 (1985).

7. J. K. Percus, J. Stat. Phys. 16:299 (1977).

8. G. Rauzy, Propriétés statistiques des suites arithmétiques (Presses Universitaires de France, 1976). 